\title{
A New Computing Method for Greeks Using Stochastic Sensitivity Analysis
}

\author{
Masato Koda \\ Systems and Information Engineering, University of Tsukuba \\ 1-1-1 Tennou-Dai, Tsukuba, 305-8573 Japan \\ koda@sk.tsukuba.ac.jp
}

\begin{abstract}
In a risk management of derivative securities, Greeks, i.e. sensitivity coefficients, are important measures of market risk to evaluate the impact of misspecification of some stochastic model on the expected payoff function. We investigate a new computing method for Greeks based on Malliavin calculus without resort to a direct differentiation of the complex payoff functions. As a result, a new relation between $\Gamma$ and $\Delta$ is obtained for the Asian option.
\end{abstract}

Keywords: Greeks, Malliavin calculus, Monte Carlo simulation.

\section{Introduction}

We consider a stochastic integral equation in a well-defined Black-Scholes set-up,

$$
S_{t}=S_{0}+\int_{0}^{t} r S_{\tau} d \tau+\int_{0}^{t} \sigma S_{\tau} d W_{\tau}
$$

where $S_{t}$ is the price of underlying asset with $S_{0}$ denoting the present (initial) value, $r$ denotes the risk-free interest rate, $\sigma$ is the volatility, and $\left(W_{t}\right)_{0 \leq t \leq T}$ is a standard Brownian motion. It should be noted that, in European options, we have a closed solution to (1) as $S_{T}=S_{0} \exp \left(\mu T+\sigma W_{T}\right)$, where $\mu=r-\sigma^{2} / 2$ for a fixed expiration or maturity time $T$.

We are interested in studying how to evaluate the sensitivity with respect to model parameters, e.g., present price $S_{0}$, volatility $\sigma$, etc., of the expected payoff

$$
E\left[e^{-r T} \Phi\left(S_{T}\right)\right]
$$

for an exponentially discounted value of the payoff function $\Phi\left(S_{T}\right)$, where $E[\cdot]$ denotes the expectation operator. The sensitivity of more sophisticated payoff functions including path-dependent Asian-type options like

$$
E\left[e^{-r T} \Phi\left(\frac{1}{T} \int_{0}^{T} S_{t} d t\right)\right]
$$

be treated in a similar manner along the lines that are investigated in this study.

In finance, this is the so-called model risk problem. Commonly referred to as Greeks, sensitivities in financial market are typically defined as the partial 
derivatives of the expected payoff function with respect to underlying model parameters. In general, finite difference approximations are heavily used to simulate Greeks. However, the approximation soon becomes inefficient particularly when payoff functions are complex and discontinuous.

To overcome this difficulty, Broadie and Glasserman [1 proposed a method to put the differential of the payoff function inside the expectation operator required to evaluate the sensitivity. But this idea is applicable only when the density of the random variable involved is explicitly known. Recently, Fournie et al. 2] suggested the use of Malliavin calculus, by means of integration by parts, to shift the differential operator from the expected payoff to the underlying diffusion (e.g., Gaussian) kernel, introducing a weighting function.

Another examples that are similar to the present study and explored by the present author (e.g., Ref. [3], [7]) but not covered in this paper are models involving a step function and non-smooth objective functions. In these studies, the stochastic sensitivity analysis technique based on the Novikov's identity is used instead of Malliavin calculus.

In this paper, we present a new constructive approach for the computation of Greeks in financial engineering. The present approach enables the simulation of Greeks without resort to direct differentiation of the complex or discontinuous payoff functions.

\section{Malliavin Calculus}

Let $R$ be the space of random variables of the form $F=f\left(W_{t_{1}}, W_{t_{2}}, \cdots, W_{t_{n}}\right)$, where $f$ is smooth and $W_{t}$ denotes the Brownian motion [6]. For a smooth random variable $F \in R$, we can define its derivative $D F=D_{t} F$, where the differential operator $D$ is closable. Since $D$ operates on random variables by differentiating functions in the form of partial derivatives, it shares the familiar chain rule property, $D_{t}(f(F))=\nabla f(F) \cdot D_{t} F=f^{\prime}(F) D_{t} F$, and others.

We denote by $D^{*}$ the Skorohod integral, defined as the adjoint operator of $D$. If $u$ belongs to $\operatorname{Dom}\left(D^{*}\right)$, then $D^{*}(u)$ is characterized by the following integration by parts (ibp) formula:

$$
E\left[F D^{*}(u)\right]=E\left[\int_{0}^{T}\left(D_{t} F\right) u_{t} d t\right]
$$

Note that (4) gives a duality relationship to link operators $D$ and $D^{*}$. The adjoint operator $D^{*}$ behaves like a stochastic integral. In fact, if $u_{t}$ is an adapted process, then the Skorohod integral coincides with the Ito integral: i.e., $D^{*}(u)=$ $\int_{0}^{T} u_{t} d W_{t}$. In general, one has

$$
D^{*}(F u)=F D^{*}(u)-\int_{0}^{T}\left(D_{t} F\right) u_{t} d t
$$

A heuristic derivation of (15) is demonstrated here. Let us assume that $F$ and $G$ are any two smooth random variables, and $u_{t}$ a generic process, then by product rule of $D$ one has 


$$
\begin{aligned}
E\left[G F D^{*}(u)\right] & =E\left[\int_{0}^{T} D_{t}(G F) u_{t} d t\right]=E\left[\int_{0}^{T} G\left(D_{t} F\right) u_{t} d t\right]+E\left[\int_{0}^{T}\left(D_{t} G\right) F u_{t} d t\right] \\
& =E\left[G \int_{0}^{T}\left(D_{t} F\right) u_{t} d t\right]+E\left[G D^{*}(F u)\right]
\end{aligned}
$$

which implies that $E\left[G D^{*}(F u)\right]=E\left[G\left(F D^{*}(u)-\int_{0}^{T}\left(D_{t} F\right) u_{t} d t\right)\right]$ for any random variables $G$. Hence, we must have (5).

In the present study, we frequently use the following formal relationship to remove the derivative from a (smooth) random function $f$ as follows:

$$
E[\nabla f(X) Y]=E\left[f^{\prime}(X) Y\right]=E\left[f(X) H_{X Y}\right],
$$

where $X, Y$, and $H_{X Y}$ are random variables. It is noted that (6) can be deduced from the integration by parts formula (4), and we have an explicit expression for $H_{X Y}$ as

$$
H_{X Y}=D^{*}\left(\frac{Y}{\int_{0}^{T} D_{t} X d t}\right) .
$$

If higher order derivatives are involved then one has to repeat the procedure (6) iteratively.

\section{European Options}

In the case of European option whose payoff function is defined by (2), the essence of the present method is that the gradient of the expected (discounted) payoff, $\nabla E\left[e^{-r T} \Phi\left(S_{T}\right)\right]$, is evaluated by putting the gradient inside the expectation, i.e., $E\left[e^{-r T} \nabla \Phi\left(S_{T}\right)\right]$, which involves computations of $\nabla \Phi\left(S_{T}\right)=\Phi^{\prime}\left(S_{T}\right)$ and $\nabla S_{T}$. Further, applying Malliavin calculus techniques, the gradient is rewritten as $E\left[e^{-r T} \Phi\left(S_{T}\right) H\right]$ for some random variable $H$. It should be noted, however, that there is no uniqueness in this representation since we can add to $H$ any random variables that are orthogonal to $S_{T}$. In general, $H$ involves Ito or Skorohod integrals.

\subsection{Delta}

Now we compute Delta, $\Delta$, the first-order partial differential sensitivity coefficient of the expected outcome of the option, i.e., (2), with respect to the initial asset value $S_{0}$ :

$$
\Delta=\frac{\partial}{\partial S_{0}} E\left[e^{-r T} \Phi\left(S_{T}\right)\right]=e^{-r T} E\left[\Phi^{\prime}\left(S_{T}\right) \frac{\partial S_{T}}{\partial S_{0}}\right]=\frac{e^{-r T}}{S_{0}} E\left[\Phi^{\prime}\left(S_{T}\right) S_{T}\right] .
$$

Then, with $X=Y=S_{T}$ in (7), we perform the integration by parts (ibp) to give

$$
\Delta=\frac{e^{-r T}}{S_{0}} E\left[\Phi\left(S_{T}\right) H_{X Y}\right]=\frac{e^{-r T}}{S_{0}} E\left[\Phi\left(S_{T}\right) D^{*}\left(\frac{S_{T}}{\int_{0}^{T} D_{t} S_{T} d t}\right)\right]
$$


Since $\int_{0}^{T} D_{t} S_{T} d t=\sigma \int_{0}^{T} S_{T} D_{t} W_{T} d t=\sigma S_{T} \int_{0}^{T} 1_{\{t \leq T\}}=\sigma T S_{T}$, we evaluate the stochastic integral

$$
H_{X Y}=D^{*}\left(\frac{S_{T}}{\int_{0}^{T} D_{t} S_{T} d t}\right)=D^{*}\left(\frac{1}{\sigma T}\right)=\frac{D^{*}(1)}{\sigma T}=\frac{W_{T}}{\sigma T}
$$

with the help of (5) applied to $u=1$ (a constant process which is adapted and Ito integral yields $\left.D^{*}(1)=W_{T}\right)$. Then the final expression for $\Delta$ reads

$$
\Delta=\frac{e^{-r T}}{\sigma T S_{0}} E\left[\Phi\left(S_{T}\right) W_{T}\right]
$$

\subsection{Vega}

Next Greek Vega, $V$, is the sensitivity of (2) with respect to the volatility $\sigma$ :

$$
V=\frac{\partial}{\partial \sigma} E\left[e^{-r T} \Phi\left(S_{T}\right)\right]=e^{-r T} E\left[\Phi^{\prime}\left(S_{T}\right) \frac{\partial S_{T}}{\partial \sigma}\right]=e^{-r T} E\left[\Phi^{\prime}\left(S_{T}\right) S_{T}\left\{W_{T}-\sigma T\right\}\right] .
$$

Then, utilizing (6) and (7) again with $X=S_{T}$ and $Y=S_{T}\left(W_{T}-\sigma T\right)$, we apply the ibp to give

$$
V=e^{-r T} E\left[\Phi\left(S_{T}\right) H_{X Y}\right] e^{-r T} E\left[\Phi\left(S_{t}\right) D^{*}\left(\frac{S_{T}\left(W_{T}-\sigma T\right)}{\int_{0}^{T} D_{t} S_{T} d t}\right)\right]=e^{-r T} E\left[\Phi\left(S_{T}\right) D^{*}\left(\frac{W_{T}}{\sigma T}-1\right)\right] .
$$

So, we evaluate the stochastic integral as

$$
H_{X Y}=D^{*}\left(\frac{W_{T}}{\sigma T}-1\right)=\frac{1}{\sigma T} D^{*}\left(W_{T}\right)-D^{*}(1)=\frac{1}{\sigma T} D^{*}\left(W_{T}\right)-W_{T} .
$$

With the help of (4) applied to $u=1$ (adapted process) and $F=W_{T}$, we have

$$
D^{*}\left(W_{T}\right)=W_{T}^{2}-\int_{0}^{T} D_{t} W_{T} d t=W_{T}^{2}-\int_{0}^{T} d t=W_{T}^{2}-T .
$$

If we bring together the partial results obtained above, we derive the final expression

$$
V=e^{-r T} E\left[\Phi\left(S_{T}\right)\left\{\frac{W_{T}^{2}}{\sigma T}-W_{T}-\frac{1}{\sigma}\right\}\right]
$$

\subsection{Gamma}

The last Greek Gamma, $\Gamma$, involves a second-order derivative,

$$
\Gamma=\frac{\partial^{2}}{\partial S_{0}^{2}} E\left[e^{-r T} \Phi\left(S_{T}\right)\right]=\frac{e^{-r T}}{S_{0}^{2}} E\left[\Phi^{\prime \prime}\left(S_{T}\right) S_{T}^{2}\right] .
$$

Utilizing (6) and (7) with $X=S_{T}$ and $Y=S_{T}^{2}$, we obtain after a first ibp

$$
\Gamma=\frac{e^{-r T}}{S_{0}^{2}} E\left[\Phi^{\prime}\left(S_{T}\right) D^{*}\left(\frac{S_{T}^{2}}{\int_{0}^{T} D_{t} S_{T} d t}\right)\right]=\frac{e^{-r T}}{S_{0}^{2}} E\left[\Phi^{\prime}\left(S_{T}\right) D^{*}\left(\frac{S_{T}}{\sigma T}\right)\right] .
$$


With the help of (5) applied to $u=1 / \sigma T$ (constant adapted process) and $F=$ $S_{T}$, we have

$$
D^{*}\left(\frac{S_{T}}{\sigma T}\right)=\frac{S_{T}}{\sigma T} D^{*}(1)-\frac{1}{\sigma T} \int_{0}^{T} D_{t} S_{T} d t=S_{T}\left(\frac{W_{T}}{\sigma T}-1\right) .
$$

Then, repeated application of (6) and (17) with $X=S_{T}$ and $Y=S_{T}\left(W_{T} / \sigma T-1\right)$, the second ibp yields

$$
\Gamma=\frac{e^{-r T}}{S_{0}^{2}} E\left[\Phi^{\prime}\left(S_{T}\right) S_{T}\left(\frac{W_{T}}{\sigma T}-1\right)\right]=\frac{e^{-r T}}{S_{0}^{2}} E\left[\Phi\left(S_{T}\right) D^{*}\left(\frac{S_{T}}{\int_{0}^{T} D_{t} S_{T} d t}\left\{\frac{W_{T}}{\sigma T}-1\right\}\right)\right] .
$$

With the help of (5) as before, we can evaluate the stochastic integral as

$$
D^{*}\left(\frac{S_{T}}{\int_{0}^{T} D_{t} S_{T} d t}\left\{\frac{W_{T}}{\sigma T}-1\right\}\right)=\frac{1}{\sigma T} D^{*}\left(\frac{W_{T}}{\sigma T}-1\right)=\frac{1}{\sigma T}\left\{\frac{W_{T}^{2}}{\sigma T}-W_{T}-\frac{1}{\sigma}\right\} .
$$

If we combine the results obtained above, the final expression becomes

$$
\Gamma=\frac{e^{-r T}}{\sigma T S_{0}^{2}} E\left[\Phi\left(S_{T}\right)\left\{\frac{W_{T}^{2}}{\sigma T}-W_{T}-\frac{1}{\sigma}\right\}\right] .
$$

Comparing (11) with (10), we find the following relationship between $V$ and $\Gamma$ :

$$
\Gamma=\frac{V}{\sigma T S_{0}^{2}}
$$

Since we have closed solutions for all the Greeks in European options, we can easily check the correctness of the above results.

\section{Asian Options}

In the case of Asian option whose payoff functional is defined by (3), the essence of the present approach is again that the gradient of the expected (discounted) payoff is rewritten as $E\left[e^{-r T} \nabla \Phi\left(\frac{1}{T} \int_{0}^{T} S_{t} d t\right)\right]=e^{-r T} E\left[\Phi\left(\frac{1}{T} \int_{0}^{T} S_{t} d t\right) H\right]$, for some random variable $H$. Different from the European options, however, we do not have a known closed solution in this case.

\subsection{Delta}

Delta in this case is given by

$$
\Delta=\frac{\partial}{\partial S_{0}} E\left[e^{-r T} \nabla \Phi\left(\frac{1}{T} \int_{0}^{T} S_{t} d t\right)\right]=\frac{e^{-r T}}{S_{0}} E\left[\Phi^{\prime}\left(\frac{1}{T} \int_{0}^{T} S_{t} d t\right) \frac{1}{T} \int_{0}^{T} S_{t} d t\right] .
$$

Utilizing (6) and (7) with $X=Y=\int_{0}^{T} S_{t} d t / T$, we may apply the ibp to give

$$
\left.\Delta=\frac{e^{-r T}}{S_{0}} E\left[\Phi\left(\frac{1}{T} \int_{0}^{T} S_{t} d t\right)\right) D^{*}\left(\frac{Y}{\int_{0}^{T} D_{t} X d t}\right)\right]=\frac{e^{-r T}}{S_{0}} E\left[\Phi\left(\frac{1}{T} \int_{0}^{T} S_{t} d t\right) D^{*}\left(\frac{\int_{0}^{T} S_{t} d t}{\sigma \int_{0}^{T} t S_{t} d t}\right)\right]
$$


where we have used the relationship $\int_{0}^{T} \int_{0}^{T} D_{v} S_{t} d v d t=\sigma \int_{0}^{T} t S_{t} d t$. With the help of (5) applied to $u=1 / \sigma$ (constant adapted process) and $F=\int_{0}^{T} S_{t} d t / \int_{0}^{T} t S_{t} d t$, we may obtain

$$
\Delta=\frac{e^{-r T}}{S_{0}} E\left[\Phi\left(\frac{1}{T} \int_{0}^{T} S_{t} d t\right)\left(\frac{1}{<T>}\left\{\frac{W_{T}}{\sigma}+\frac{<T^{2}>}{<T>}\right\}-1\right)\right],
$$

where we have used the relationship $\int_{0}^{T} \int_{0}^{T} t D_{v} S_{t} d v d t=\sigma \int_{0}^{T} t^{2} S_{t} d t$, and where we defined

$$
<T>=\frac{\int_{0}^{T} t S_{t} d t}{\int_{0}^{T} S_{t} d t} \text { and }<T^{2}>=\frac{\int_{0}^{T} t^{2} S_{t} d t}{\int_{0}^{T} S_{t} d t} .
$$

\subsection{Vega}

Vega in this case becomes

$$
\begin{aligned}
V & =\frac{\partial}{\partial \sigma} E\left[e^{-r T} \nabla \Phi\left(\frac{1}{T} \int_{0}^{T} S_{t} d t\right)\right]=e^{-r T} E\left[\Phi^{\prime}\left(\frac{1}{T} \int_{0}^{T} S_{t} d t\right) \frac{1}{T} \int_{0}^{T} \frac{\partial S_{t}}{\partial \sigma} d t\right] \\
& =e^{-r T} E\left[\Phi^{\prime}\left(\frac{1}{T} \int_{0}^{T} S_{t} d t\right) \frac{1}{T} \int_{0}^{T} S_{t}\left\{W_{t}-t \sigma\right\} d t\right] .
\end{aligned}
$$

As before, with the help of (6) and (77) applied to $X=\int_{0}^{T} S_{t} d t / T$ and $Y=$ $\int_{0}^{T} S_{t}\left(W_{t}-t \sigma\right) d t / T$, we have

$V=e^{-r T} E\left[\Phi\left(\frac{1}{T} \int_{0}^{T} S_{t} d t\right) D^{*}\left(\frac{Y}{\int_{0}^{T} D_{t} X d t}\right)\right]=e^{-r T} E\left[\Phi\left(\frac{1}{T} \int_{0}^{T} S_{t} d t\right) D^{*}\left(\frac{\int_{0}^{T} S_{t} W_{t} d t}{\sigma \int_{0}^{T} t S_{t} d t}-1\right)\right]$,

which, with the help of (5), yields the following expression

$$
V=e^{-r T} E\left[\Phi\left(\frac{1}{T} S_{t} d t\right)\left\{\frac{\int_{0}^{T} \int_{0}^{T} S_{t} W_{t} d t d W_{\tau}}{\sigma \int_{0}^{T} t S_{t} d t}+\frac{\int_{0}^{T} t^{2} S_{t} d t \int_{0}^{T} S_{t} W_{t} d t}{\left(\int_{0}^{T} t S_{t} d t\right)^{2}}-W_{T}\right\}\right] .
$$

\subsection{Gamma}

Gamma involves a second-order derivative,

$$
\Gamma=\frac{\partial^{2}}{\partial S_{0}^{2}} E\left[e^{-r T} \nabla \Phi\left(\frac{1}{T} \int_{0}^{T} S_{t} d t\right)\right]=\frac{e^{-r T}}{S_{0}^{2}} E\left[\Phi^{\prime \prime}\left(\frac{1}{T} \int_{0}^{T} S_{t} d t\right)\left(\int_{0}^{T} S_{t} d t / T\right)^{2}\right] .
$$

Application of (6) with $X=\int_{0}^{T} S_{t} d t / T$ and $Y=\left(\int_{0}^{T} S_{t} d t / T\right)^{2}$, and utilizing a close variant of (7) (see Ref. [4], [5]), i.e.,

$$
H_{X Y}=D^{*}\left(\frac{S_{t} Y}{\int_{0}^{T} S_{v} D_{v} X d v}\right)
$$

we may obtain after a first integration by parts 


$$
\begin{aligned}
\Gamma & =\frac{e^{-r T}}{S_{0}^{2}} E\left[\Phi^{\prime}\left(\frac{1}{T} \int_{0}^{T} S_{\tau} d \tau\right) D^{*}\left(\frac{S_{t} Y}{\int_{0}^{T} S_{v} D_{v} X d v}\right)\right] \\
& =\frac{e^{-r T}}{S_{0}^{2}} E\left[\Phi^{\prime}\left(\frac{1}{T} \int_{0}^{T} S_{\tau} d \tau\right) D^{*}\left(\frac{2 S_{t}}{\sigma T}\right)\right],
\end{aligned}
$$

where the relation $\int_{0}^{T} \int_{0}^{T} S_{v} D_{v} S_{t} d v d t=\sigma \int_{0}^{T} \int_{0}^{t} S_{t} S_{v} d v d t=\frac{\sigma}{2}\left(\int_{0}^{T} S_{t} d t\right)^{2}$ is used. Further, we obtain

$\Gamma=\frac{2 e^{-r T}}{\sigma T S_{0}^{2}} E\left[\Phi^{\prime}\left(\frac{1}{T} \int_{0}^{T} S_{\tau} d \tau\right) \int_{0}^{T} S_{t} d W_{t}\right]=\frac{2 e^{-r T}}{\sigma^{2} T S_{0}^{2}} E\left[\Phi^{\prime}\left(\frac{1}{T} \int_{0}^{T} S_{t} d t\right)\left(S_{T}-S_{0}-r \int_{0}^{T} S_{t} d t\right)\right]$, which involves (1).

Then, repeated application of (6) and (7) with $X=\int_{0}^{T} S_{t} d t / T$ and $Y=$ $S_{T}-S_{0}-r \int_{0}^{T} S_{t} d t$, the second integration by parts yields

$$
\begin{aligned}
\Gamma & =\frac{2 e^{-r T}}{\sigma^{2} S_{0}^{2}} E\left[\Phi\left(\frac{1}{T} \int_{0}^{T} S_{t} d t\right) D^{*}\left(\frac{S_{T}-S_{0}-r \int_{0}^{T} S_{t} d t}{\sigma \int_{0}^{T} t S_{t} d t}\right)\right] \\
& =\frac{2 e^{-r T}}{\sigma^{2} S_{0}^{2}} E\left[\Phi\left(\frac{1}{T} \int_{0}^{T} S_{t} d t\right) D^{*}\left(\frac{S_{T}-S_{0}}{\sigma \int_{0}^{T} t S_{t} d t}\right)\right]-\frac{2 r e^{-r T}}{\sigma^{2} S_{0}^{2}} E\left[\Phi\left(\frac{1}{T} \int_{0}^{T} S_{t} d t\right) D^{*}\left(\frac{\int_{0}^{T} S_{t} d t}{\sigma \int_{0}^{T} t S_{t} d t}\right)\right] .
\end{aligned}
$$

With the help of (5) applied to $u=1 / \sigma$ and $F=\left(S_{T}-S_{0}\right) / \int_{0}^{T} t S_{t} d t$, the present approach yields a brand new estimate which gives an explicit relationship between $\Gamma$ and $\Delta$ as follows:

$$
\begin{aligned}
\Gamma= & \frac{2 e^{-r T}}{\sigma^{2} S_{0}^{2}} E\left[\Phi\left(\frac{1}{T} \int_{0}^{T} S_{t} d t\right) D^{*}\left(\frac{S_{T}-S_{0}}{\sigma \int_{0}^{T} t S_{t} d t}\right)\right]-\frac{2 r}{\sigma^{2} S_{0}} \Delta \\
= & \frac{2 e^{-r T}}{\sigma^{2} S_{0}^{2}} E\left[\Phi\left(\frac{1}{T} \int_{0}^{T} S_{t} d t\right) \frac{1}{\int_{0}^{T} t S_{t} d t}\left(\left\{\frac{W_{T}}{\sigma}+\frac{<T^{2}>}{<T>}\right\}\left(S_{T}-S_{0}\right)-T S_{T}\right)\right] \\
& -\frac{2 r}{\sigma^{2} S_{0}} \Delta .
\end{aligned}
$$

\section{Monte Carlo Simulations of Asian Option}

Here, we present the simulation results with parameters $r=0.1, \sigma=0.25$, $T=0.2$ (in years), and $S_{0}=K=100$ (in arbitrary cash units) where $K$ denotes the strike price. We have divided the entire interval of integration into 252 pieces, representing the trading days in a year.

In Fig. 1, we compare the convergence behavior of $\Delta$ with the result obtained by Broadie and Glasserman [1]. The result indicates a fairy good convergence to the steady-state value that is attained at 10,000th iteration stage in [1].

In Fig. 2, we compare the simulation result of $V$ with the one that is obtained at 10,000th iteration stage in 1. This indicates that some noticeable bias may remain in the present Monte Carlo simulation, and further study may be necessary to analyze and reduce the bias involved.

Although we cannot compare the proposed method with others, the present simulations may provide most detailed and extensive results currently available for Greeks of Asian option. 


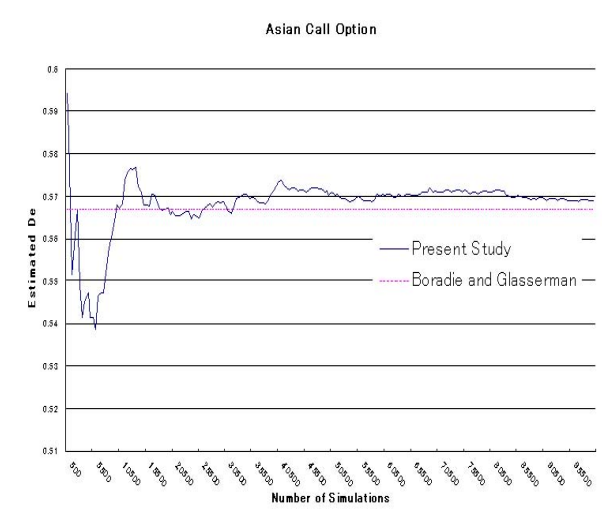

Fig. 1. Estimated Delta

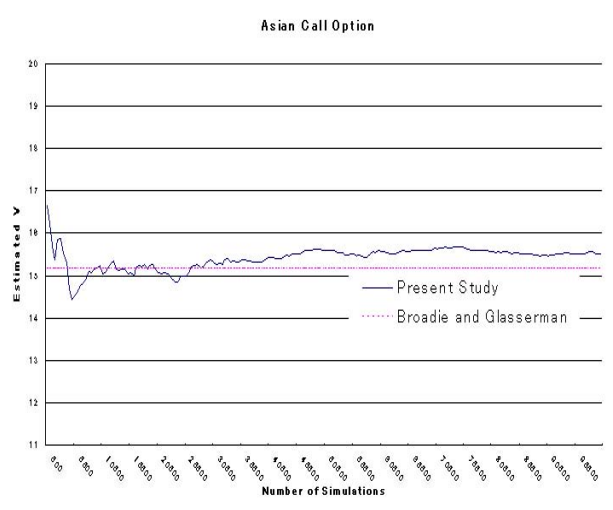

Fig. 2. Estimated Vega

\section{Conclusions}

We have presented a stochastic sensitivity analysis method, in particular, a constructive approach for computing Greeks in finance using Malliavin calculus. As a result, a new relation between $\Gamma$ and $\Delta$ is obtained for the Asian option. The present approach may be useful when the random variables are smooth in the sense of stochastic derivatives. It is, however, necessary to further investigate and improve Monte Carlo procedures to reduce the bias involved in the simulation of Vega in Asian-type options and other sophisticated options.

\section{References}

1. Broadie, M., Glasserman, P.: Estimating security price derivatives using simulation. Management Science 42 (1996) 269-285

2. Fournie, E., Lasry, J.M., Lebuchoux, L., Lions, P.L.: An application of Malliavin calculus to Monte Carlo methods in Finance II. Finance and Stochastics 5 (2001) 201-236

3. Koda, M., Okano, H.: A new stochastic learning algorithm for neural networks. Journal of the Operations Research Society of Japan 43 (2000) 469-485

4. Koda, M., Kohatsu-Higa, A., Montero, M.: An Application of Stochastic Sensitivity Analysis to Financial Engineering. Discussion Paper Series, No. 980, Institute of Policy and Planning Sciences, University of Tsukuba (2002)

5. Montero, M., Kohatsu-Higa, A.: Malliavin calculus applied to finance. Physica A 320 (2003) 548-570

6. Nualert, D.: The Malliavin Calculus and Related Topics. Springer, New York (1995)

7. Okano, H., Koda, M.: An optimization algorithm based on stochastic sensitivity analysis for noisy objective landscapes. Reliability Engineering and System Safety, 79 (2003) 245-252 